\title{
Psychological stress enhances keloid development via stress hormone-induced abnormal cytokine profiles and inflammatory responses
}

\author{
Ya-Ting Yang, Xiao-Li Wu\#, Wei Liü \\ Department of Plastic and Reconstructive Surgery, Shanghai Tissue Engineering Key Laboratory, Shanghai Research Institute of \\ Plastic and Reconstructive Surgery, Shanghai 9th People's Hospital, Shanghai Jiao Tong University School of Medicine, Shanghai \\ 200011, China. \\ \#These authors contributed equally to this work.
}

Correspondence to: Dr. Wei Liu and Dr. Xiao-Li Wu, Department of Plastic and Reconstructive Surgery, Shanghai Tissue Engineering Key Laboratory, Shanghai Research Institute of Plastic and Reconstructive Surgery, Shanghai 9th People's Hospital, Shanghai Jiao Tong University School of Medicine, 639 Zhi Zao Ju Rd, Shanghai 200011, China.

E-mail: liuwei_2000@yahoo.com; wuxiaoli528@icloud.com

How to cite this article: Yang YT, Wu XL, Liu W. Psychological stress enhances keloid development via stress hormone-induced abnormal cytokine profiles and inflammatory responses. Plast Aesthet Res 2020;7:34.

http://dx.doi.org/10.20517/2347-9264.2020.24

Received: 17 Feb 2020 First Decision: 27 Apr 2020 Revised: 17 Jun 2020 Accepted: 20 Jun 2020 Available online: 12 Jul 2020

Academic Editor: Alexis Desmoulière, Jérôme Laloze Copy Editor: Cai-Hong Wang Production Editor: Jing Yu

\begin{abstract}
Keloid is a fibroproliferative disorder resulting from the abnormal wound healing process, and it causes both cosmetic concerns and functional disabilities. Genetic predisposition, wound trauma, foreign body reaction, mechanical stretch, and immune dysfunction are common risk factors, but there remain mechanisms unclarified, leaving challenges in addressing the clinical concerns of recurrence and resistance. However, similar patterns of growth and metabolism between keloids and cancers provide a unique insight into the future exploration of keloid pathogenesis. Psychological stress has been demonstrated to be involved in the development and drug resistance of multiple cancers, but this aspect remains less-explored in keloids. Clinical observations and published investigations have noticed that persistent stress is common among keloid patients and their symptoms tend to deteriorate under stressful conditions. Following a thorough review of the published literature, we have identified three signaling pathways that might imply how stress hormones are likely to influence the keloid pathogenesis via activating adrenergic receptors and dysregulating the immune system. Thus, we hypothesized that psychological stress would be a key risk factor for keloid development via stimulating fibrosis, aggravating local hypoxia, and inflammation.
\end{abstract}

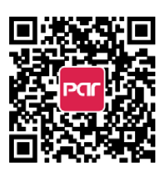


Keywords: Keloids, psychological stress, adrenergic receptors, dysregulated immune system

\section{INTRODUCTION}

Keloids give rise to both cosmetic concerns and functional disabilities as a result of fibroproliferative disorder and excessive collagen deposition after abnormal wound healing. Furthermore, subjective symptoms derived from this disease such as pain and pruritus could dramatically affect patients' quality of life by causing significant psychological stress. Previous studies have revealed that the genetic background, wound trauma, foreign body reaction, mechanical stretch, and immune dysfunction are critical risk factors for keloid development, but the exact mechanism of keloid formation could be more complicated than what has been found and other risk factors are also likely to be involved, posing challenges to clinical treatment.

Described as "the non-specific response of the body" ${ }^{[1]}$, psychological stress has a complicated and profound influence on the functional state of affected human bodies by secreting various stress hormones. These primarily include glucocorticoids through the activation of the hypothalamic-pituitary-adrenal axis and catecholamines through the sympathetic nervous system ${ }^{[2]}$. Glucocorticoids can significantly affect cell metabolism and immune functions in the long term, while the effects of catecholamines [norepinephrine (NE), epinephrine (E)] are mediated via binding to $\alpha$-adrenergic receptors $(\alpha$-AR) or $\beta$-adrenergic receptors ( $\beta$-ARs) facilitating the human body to react to all kinds of stressors. However, if the stressful situation becomes overwhelming, the combined action of stress stimulators remains persistent the physiologically maintained balance maybe disrupted leading to enhancement of pathophysiological processes for multiple diseases.

Psychological stress has been indicated for contributing to cancer development for decades. For example, in patients with lung cancers, stress has become an established predictor of mortality ${ }^{[3]}$. Stress hormones (NE, E) can also promote resistance to tyrosine kinase inhibitors (TKIs) of epidermal growth factor receptor (EGFR) in non-small cell lung cancer (NSCLC) ${ }^{[4]}$ and increase tumor-derived interleukin-6 (IL-6) overexpression in ovarian cancer cells ${ }^{[5]}$. Featured with uncontrollable proliferation, invasiveness, and glycolysis-dominant metabolic pattern ${ }^{[6]}$, keloids are regarded as benign skin tumors and the accumulating literature evidence suggests that certain pathogenic signaling pathways might be shared between keloids and tumors. As an example, the elevation of IL- 6 level, which was determined as a promoting agent in NSCLC resistance and its poor prognosis, has also been identified to contribute to keloid formation ${ }^{[7]}$. Therefore, it would be reasonable to investigate whether psychological stress influences keloid pathogenesis and explore the potential of stress hormones (NE, E) as therapeutic targets.

The psychological and mental impacts of pathological scars have been studied in clinical settings and been reported in an investigation among a black African population. Of this, $48.9 \%$ of keloid patients thought they were stigmatized and $35.8 \%$ complained about their limited social interactions ${ }^{[8]}$. Furtado et al.$^{[9]}$ from Brazil have reported psychological stress as a risk factor for postoperative keloid recurrence in a clinical study and proposed a novel psycho-neuro-immune-endocrine etiology where they pictured a macroscope of the "brain-skin connection" in keloid pathogenesis without mentioning detailed pathways ${ }^{[10]}$. For quite a long time, the potential association between stress hormones and keloid pathogenesis has been neglected according to Pubmed and Embase database searched with key words: keloid AND stress, keloid AND psychological stress, keloid AND mood disorders. By analyzing the published evidence with regards to bio-active molecules in keloid tissues and the effects of stress hormones (NE, E) on skin fibroblasts and immune cells (macrophages) in vitro and immune cytokine profiles both in vitro and in vivo, we outline in this manuscript three possible signaling pathways that might explain these phenomena. 


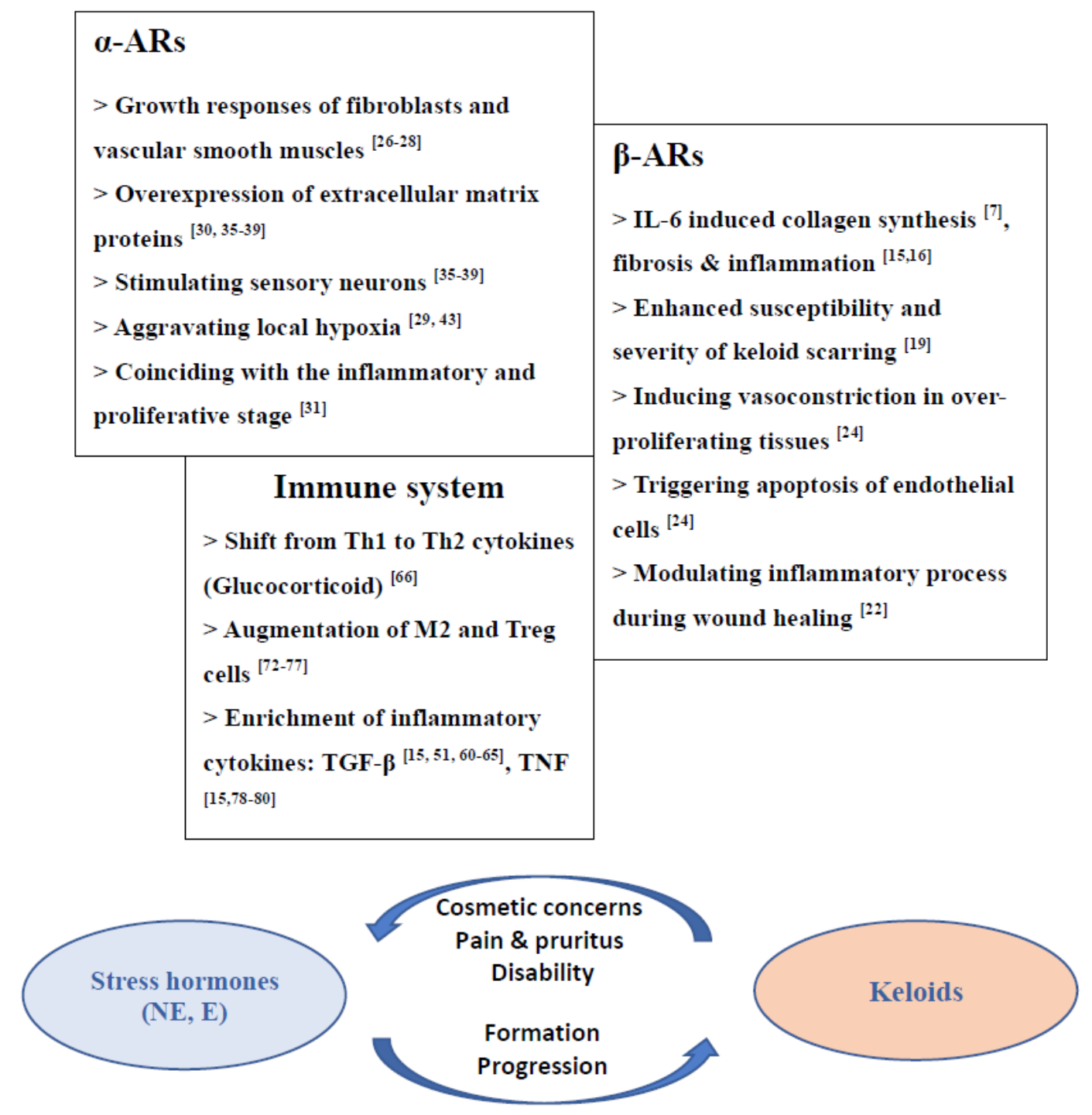

Figure 1. Three possible pathways that may explain how stress hormones (NE, E) could induce abnormal cytokine profiles and inflammatory responses. TGF: transforming growth factor; TNF: tumor necrosis factor; AR: adrenergic receptors; IL: interleukin; NE: norepinephrine; E: epinephrine

\section{HYPOTHESIS}

The psychological and mental state of keloid patients has been investigated and observed in clinical practice, but unfortunately, the association between the psychological stress-induced pathological alterations in keloids has been neglected. Based on the published literature, we propose the hypothesis that psychological stress can be a risk factor of keloid development as stress hormones (NE, E) might contribute to keloid pathogenesis [Figure 1]. Therefore, attenuating the AR-receptor function(s) may enhance the efficacy of traditional keloid treatments and reduce the therapeutic resistance. The following sections provide a detailed description of the hypothesis with related supporting evidence from the literature.

\section{EVALUATIONS OF THE HYPOTHESIS: THE PROMOTING EFFECT OF PSYCHOLOGICAL STRESS ON KELOID PATHOGENESIS}

Stress hormones could increase IL- 6 expression to enhance fibrosis via activating $\beta$-ARs As a critical mediator of fibrosis ${ }^{[11-14]}$ and inflammation ${ }^{[15]}$, elevated IL- 6 level has been identified in both keloid tissues and psychologically stressed population. Other studies revealed that the stress hormones 
(NE, E) increased IL- 6 expression and increased resistance in NSCLC patients ${ }^{[4]}$. Therefore, we hypothesize that the psychological stress (NE, E) might stimulate keloid pathogenesis by enhancing IL-6 expression via activating $\beta$-ARs, and the use of $\beta$-blockers such as propranolol might facilitate the efficacy of current keloid treatments.

IL- 6 is one of the Th1 type cytokines related to pro-fibrosis and inflammation ${ }^{[16,17]}$, which has been regarded as a marker of keloid progression. A significant increase of IL- 6 and IL- 6 signaling elements was observed in keloid fibroblasts (KFs) compared to normal fibroblasts (NFs) ${ }^{[7]}$. Moreover, the induction of IL-6 by IL- 6 peptide in NF cultures or inhibition of IL- 6 or IL- 6 Ra by their corresponding antibodies in KF cultures rendered a dose-dependent increase or decrease in the synthesis of collagen type $\mathrm{I}^{[7]}$. This was possibly the result of the suppression of matrix metalloproteinases (MMPs) at mRNA level and pro-matrix MMPs at the protein level ${ }^{[18]}$. Besides, a Japanese population-based study involving IL6R genotypic and allelic analyses among 239 normal and 376 keloid patients revealed that the IL-6 572G/C polymorphism is associated with susceptibility to keloid formation and the severity of keloid scarring ${ }^{[19]}$.

Multiple stress models have confirmed that a higher plasma level of IL-6 was also observed in people with depressive symptoms or at a stressed state (for example, angry couples after a domestic conflict or vaccination with an influenza virus vaccine $)^{[20,21]}$, suggesting a stress hormone (NE, E)-mediated IL-6 augmentation.

The importance of psycho-physiological interactions has gained increasing attention recently, and NEinduced IL- 6 elevation has been taken as a predictor of treatment resistance and poor outcomes in certain cancers. For example, researchers found that by binding to $\beta 2$-ARs, stress hormones (NE, E) can subsequently induce IL- 6 expression via suppressing liver kinase B1 and activating cAMP-responsive element-binding protein. Therefore, Combinational treatments with propranolol ( $\beta$-AR inhibitor) could effectively lower the IL-6 concentration and prolong the progression-free survival in EGFR TKIs resistant patients ${ }^{[4]}$. Apart from lung cancers, it was also observed that the NE/E induced activation of ARs also resulted in a similar increase of IL-6 in ovarian cancer cells ${ }^{[5]}$.

The $\beta$-ARs have been suggested as potential pharmacologic targets of catecholamine actions that influence numerous physiological and metabolic activities systemically in human bodies ${ }^{[22,23]}$. Both in vitro and in vivo studies of $\beta$-ARs carried out over the past decades focused mainly on their effects on cardiac function, whereas reported studies on non-cardiac $\beta$-blocker effects focused on their roles in the wound healing process $^{[22]}$. Propranolol is a representative nonselective $\beta$-adrenergic blockade agent with promising efficacy in rhythm disturbances and hypertension. It was found that in propranolol-treated animals, wound contraction and the formation of the neo-epidermis and granulation tissue were delayed ${ }^{[22]}$. de Mesquita ${ }^{[24]}$ hypothesized that systemic or intralesional injection of propranolol could serve as a novel cure of keloids because of its potential to induce vasoconstriction in over-proliferating tissues, trigger apoptosis of endothelial cells, and modulate inflammatory process during wound healing. Moreover, one singleinstitution case-control study in 2017 also observed better scar formation in post-surgery patients who were administrated with $\beta$ blockers ${ }^{[25]}$. It is noteworthy that patients with abnormal scar histories or family tendency are excluded in this study, and the administration of other hypertension drugs such as calcium channel blockers showed no association with the scar quality, a phenomenon that indirectly supports our hypothesis that adrenergic activation might be an independent risk factor for the pathogenesis of keloid and hypertrophic scars ${ }^{[25]}$. Noticeably, yearlong administration of oxandrolone and propranolol successfully reduced scar severity and pliability in the -post-burn hypertrophic scar patients and their emotional health state was also improved ${ }^{[26]}$. Encouraged by the findings of the propranolol-based study showingthe reduction in NE-induced IL- 6 elevation with an altered prognosis of NSCLC patients and the studies outlined above, we strongly propose that it might be promising to use propranolol for targeting $\beta$-ARs on keloid cells to disrupt IL-6 mediated keloid pathogenesis. 


\section{Stress hormones could activate $\alpha$-ARs to promote keloid formation by enhancing growth- related responses and aggravating local hypoxia environment}

The $\alpha$-ARs are another group of adrenergic receptors that were found to be increased in keloid tissues and peripheral sensory neurons of scarred skin. Stress hormones could activate $\alpha$-ARs to promote keloid formation by enhancing growth-related responses and aggravating the local hypoxia environment. Furthermore, their enhanced expression was associated with cell proliferation, inflammation, and uncomfortable symptoms of pain and pruritus in hypertrophic scars.

Activated $\alpha$-ARs can evoke growth-related responses after bonding to stress hormones (NE, E). For example, the $\alpha-1 \mathrm{~B}$ subtype stimulates cell proliferation ${ }^{[27-29]}$ and the activation of the $\alpha-1 \mathrm{~A}$ subtype evokes protein biosynthesis and cell hypertrophy ${ }^{[30]}$. Tissue biopsy and immunohistochemistry detected an increase of $\alpha$-ARs in keloid scars compared to burn scars and unscarred skins in the regenerated epidermis, dense bands of cells in the upper dermis and collagen fibers in the deep dermis, coinciding with the inflammatory and proliferative stage ${ }^{[31]}$. Since it was reported that injury-induced growth-related responses are $\alpha 1$-AR subtype-dependent ${ }^{[32-34]}$, over-expression of $\alpha 1$-ARs might result in both hyperplasia and hypertrophy of fibroblasts and vascular smooth muscles in keloid tissues. Moreover, the adrenergic activation of fibroblasts could increase the production of extracellular matrix proteins (such as collagen and fibronectin) and the expression of $\alpha 1$-ARs in peripheral sensory neurons was in line with enhanced sensitivity to adrenergic agents in injured tissues, suggesting that the up-regulation of $\alpha 1$-ARs might not only be involved in the inflammation and wound healing processes, but also be a significant source of pain, itching, and hyperaesthesia ${ }^{[31]}$.

Apart from $\alpha 1$-AR-induced growth-related responses that have been discussed, the vasoconstriction caused by activated $\alpha 1$-ARs in vascular smooth muscles is another important aspect that could aggravate the local hypoxia of keloid microenvironment and trigger hypoxia-related pathogenesis. Hypoxia is a common environmental stress factor associated with various physiological and pathological conditions, including angiogenesis, cell proliferation, glucose metabolism, $\mathrm{pH}$ regulation, and migration ${ }^{[35,36]}$. Accumulating evidence suggested an anoxic microenvironment is crucial in keloid pathogenesis because of abnormal hypoxia-associated occluded microvessels, which is also partially responsible for keloid resistance to radiation therapy ${ }^{[37]}$. It was observed that the central area of keloid is severely ischemic, exhibiting higher hypoxia-inducible factor-1a (HIF-1a) expression and lower vascular density than their marginal areas and normal skin borders ${ }^{[38,39]}$. The HIF- $1 \alpha$ is also involved in the inflammatory process by regulating angiogenesis and inflammatory cell functions ${ }^{[40-42]}$. Blocking HIF-1 signal pathways by either 2 ME2 or HIF-1 $\alpha$ siRNA has been shown to successfully increase the radiation-induced apoptosis in keloid fibroblasts ${ }^{[37]}$. Hypoxia can also drive the transition of human dermal fibroblasts to a myofibroblast-like phenotype via the transforming growth factor $-\beta 1 / \mathrm{SMAD} 3$ pathway ${ }^{[36]}$, and increase the expression of vascular endothelial growth factor (VEGF) in keloids ${ }^{[35,38]}$. Glycolysis, the major glucose metabolic pattern for keloid tissues, could also interact with hypoxia and promote the lactate accumulation, resulting in excessive collagen production and fibrogenic activities ${ }^{[43]}$.

\section{Stress hormones might influence keloid formation by dysregulating the immune system and inflammation}

The classical model of wound healing involves three distinct but overlapping phases that chronologically occur as the inflammatory, the proliferative, and the remodeling phases. Disturbance of these processes, especially the prolonged and excessive inflammatory reactions could lead to an increase of fibroblast activities and excessive extracellular matrix $(\mathrm{ECM})$ production $^{[44]}$. The available evidence indicates that malfunction of the immune system and inflammation might be involved in keloid formation. Keloid tissues are highly infiltrated with various immune cells, immunoglobulins and complements ${ }^{[45]}$, as well as growth factors, cytokines and proteases, such as IL-6, tumor necrosis factor (TNF), transforming growth 
factor- $\beta$ (TGF- $\beta$ ), platelet-derived growth factor (PDGF) and epidermal growth factor (EGF) ${ }^{[46,47]}$, which are critical for the migration, proliferation and collagen synthesis of fibroblasts. Moreover, previous studies have demonstrated that the expression of several immune-related genes was also dysregulated in keloid tissues $^{[48,49]}$.

The elevated C-reactive protein (CRP, a marker of inflammation) plasma concentration in caregivers of Alzheimer's patients ${ }^{[50,51]}$ also implied that psychological stress was a potent inducer of the chronic inflammation state. Analysis of stress models revealed that stress hormones (NE, E, glucocorticoid) have detrimental effects on immune functions as well as the inflammation process from various aspects ${ }^{[52]}$. Here are some keloid-associated inflammatory molecules or mechanisms that have been identified dysregulated in stressed conditions.

\section{TGF- $\beta$}

TGF- $\beta$ is probably the most fibrogenic factor associated with keloid formation by acting as a strong chemotactic agent for fibroblasts ${ }^{[53]}$ and increasing cell rigidity through TGF- $\beta 1$ receptor-smooth muscle actin axis $^{[54]}$. It is also known as a regulatory resolution factor that can induce remodeling within sites of damaged tissues upon mood disorder-associated inflammatory processes ${ }^{[15]}$.

In the inflammatory and proliferative phase, degranulation of platelets releases and activates several fibrogenic growth factors and chemotactic agents including TGF- $\beta 1$ and TGF- $\beta 2^{[44]}$, to increase the corresponding receptors and responsiveness compared to fibroblasts from normal tissues ${ }^{[55-58]}$. Although there is no direct evidence that stress hormones can enhance the efficacy of TGF- $\beta$ in keloids, it is nevertheless clear that TGF- $\beta$ plays a pivotal role in keloid formation and stress-derived inflammatory conditions.

\section{The cells and cytokines}

CD4 $\mathrm{T}$ cells express $\mathrm{T}$ helper lymphocyte (Th)1 or Th2 responses, while glucocorticoids are thought to cause a shift from Th1 to Th2 cytokines by downregulating Th1 cytokines and upregulating Th2 cytokines $^{[59]}$. Th1 responses produce interferons and IL-12 and are thought to be related to the attenuation of fibrogenesis, whereas Th2 responses (IL-4, IL-5, IL-10 and IL-13. IL-1, and IL-6) are generally related to fibrogenesis, among which IL-4, IL-5, IL-6, and IL-13 are thought to be essential for promoting fibroblast recruitment and proliferation, ECM deposition, angiogenesis and re-epithelialization ${ }^{[16,17,47]}$ (except for IL-10, which are mainly related to anti-fibrosis ${ }^{[60-62]}$. In a published report, stress was associated with a decrease in IL-2 receptor (IL-2R) mRNA levels and the protein expression in peripheral blood leukocytes compared to the baseline ${ }^{[63]}$. In a longitudinal study over 6 years, caregivers and former caregiver's (a kind of stress model) showed elevated plasma IL- 6 levels that increased at a rate four times faster than those of age-matched controls ${ }^{[50]}$. Elevation of serum IL-6 (a marker of inflammation) levels have been previously described in both chronically stressed older adults ${ }^{[6]}$ and keloid patients. Since a clear NE-IL-6 pathway has been identified in NSCLCs and ovarian cancer ${ }^{[4,5]}$, we presumed that a similar NE-induced IL-6 elevation might exist in keloid. Stress hormones and related receptors could thus serve as feasible therapeutic targets.

\section{Macrophages and treg cells}

Macrophages are divided into two subsets, the IL-12- and inducible nitric oxide synthase (iNOS)-expressing M1 type and the IL-10- and TGF- $\beta$-expressing M2 type ${ }^{[65]}$. The classically-activated (M1) cells that secrete pro-inflammatory cytokines, whereas alternatively-activated (M2) cells that foster tissue repair and regeneration ${ }^{[6,6]}$. It was found that $\mathrm{M} 1$-associated genes, including iNOS and IL-12, were less elevated in keloid tissues than $\mathrm{M} 2$-associated genes, including IL-10 and TGF- $\beta^{[68]}$, suggesting that macrophages in keloids were shifted toward the M2 polarization. 
The Foxp ${ }^{+} \mathrm{CD}^{+}$regulatory $\mathrm{T}$ (Treg) cells represent a critical $\mathrm{T}$ cell subset, the dysfunction of which was implicated in multiple inflammatory diseases ${ }^{[69]}$. While the normal skin displays a relative lack of $\mathrm{CD}^{+} \mathrm{T}$ cells, the levels of Foxp $3^{+}$Treg were significantly higher in keloid tissues (range of 25.5\%-72.5\%) in contrast to those in the circulation of keloid patients $(4 \%-10.5 \%)^{[68]}$. Additionally, it was also observed in the same research that incubating circulating $\mathrm{CD}_{3}{ }^{+} \mathrm{T}$ cells with keloid macrophages could significantly raise the expression of Foxp3, suggesting that these keloid macrophages could promote Treg differentiation by upregulating Foxp3 expression.

Even though no research on the levels of macrophages and depression in patients with skin conditions has been reported thus far, other previous studies did show that the activation and polarization of microglia (central nervous system-resident macrophages) could modulate the production and secretion of proinflammatory cytokines, implicating the involvement of macrophages in the etiology of major depressive disorder, which was referred as the "macrophage theory of depression" ${ }^{\text {"[70] }}$. In light of this, it could be argued that an abundance of pro-inflammatory cytokines induced by the altered profile of macrophages inside keloids might exist, and further investigations, therefore, need to be performed in keloid patients with diagnosed depression.

\section{The tumor necrosis factor}

TNF produced by monocytes and macrophages during the inflammatory phase has been known to induce collagen degranulation and minimize excessive scarring possibly by increasing the MMP1/TIMP3, MMP2/ TIMP3 ratios $^{[71]}$.

Various animal and patient-based clinical studies have demonstrated the associations between the concentration of pro-inflammatory cytokines, specifically IL-1 $\beta$, IL-6, TNF, and depressive symptoms ${ }^{[15]}$. They also showed a general normalization (decline) of IL- 6 and TNF concentration after antidepressant treatment ${ }^{[72,73]}$.

\section{CONCLUSION}

As a benign skin tumor outgrowing the original wound boundary or growing spontaneously on the normal skin, keloid can bring great pain and inconvenience to patients. Although risk factors such as genes and infection have been noticed, the pathologic mechanisms remain unclear, leading to challenges for treatment resistance and keloid recurrence, including 9\%-50\% recurrent rate of the corticosteroid injection, $45 \%-100 \%$ recurrence rate of the surgical removal and $9.59 \%$ relapse rate of the radiotherapy ${ }^{[4]}$.

Psychological stress evoked by traumatic events and depressive conditions has huge impacts on the overall health state. Through stress hormones (NE, E, glucocorticoids) and their respective receptors, tissuespecific responses are triggered as well as the general modulation of the immune system and inflammation. Studies have confirmed that stress hormones are critical for the initiation and development of multiple diseases $^{[2,3,52]}$, but the impact of psychological stress on keloid pathogenesis has been neglected.

As for keloid patients, the original trauma, uncomfortable feelings, together with the cosmetic concerns are all potent and constant underlying stressors, which make them very likely to be trapped in a stressintensive state. Therefore, psychological stress is a pivotal and inevitable element that should be taken into consideration in formulating optimal treatment regimens. In view of the reviewed literature, especially with regards to stress hormone-induced cellular and physiological changes observed during psychological stress, we advance the hypothesis that stress hormones (NE, E) may participate in the keloid formation by: (1) increasing the expression of keloid-associated IL-6 via activating $\beta$-ARs; (2) triggering growth responses of fibroblasts and symptoms(pain and pruritus) in scar tissues by directly activating $\alpha$-ARs; (3) exacerbating 
the local hypoxia conditions; and (4) dysregulating immune systems to provide an inflammatory microenvironment that is in favor of keloid formation, thus promoting keloid pathogenesis.

Although the impact of psychological stress on the pathogenesis of diseases, such as cancers, has been known for decades, its relevance and impact concerning keloid pathogenesis and therapy have barely been studied. Although the stressed state in keloid patients has been well-observed during clinical practice and demonstrated by investigations in an African population, more direct evidence are required in the future. For example, current studies have detected elevated adrenergic receptors, but the concentration and distribution of catecholamines in keloid tissues remain uninvestigated, so are specific cytokines and immune cells in the targeted population. Clinically, large-scale evaluations of psychological stress among keloid patients should also be undertaken as a follow up to "as a proof of principle" pilot studies. Moreover, studies combing $\alpha, \beta$-receptor antagonists and anti-depressant medicines with conventional keloid therapies could be explored in future clinical trials to realize better treatment outcomes.

\section{DECLARATIONS}

\section{Author's contributions}

Made substantial contributions to the conception of the hypothesis: Yang YT, Wu XL, Liu W

\section{Availability of data and materials}

Not applicable.

\section{Financial support and sponsorship}

This work is financially supported by the National Natural Science Foundation (81671921).

\section{Conflict of interest}

All authors declared that there are no conflicts of interest.

\section{Ethical approval and consent to participate}

Not applicable.

\section{Consent for publication}

Not applicable.

\section{Copyright}

(c) The Author(s) 2020.

\section{REFERENCES}

1 Selye H. Stress without distress. Le stress sans detresse. Brux Med 1976;56:205-10.

2. Kyrou I, Tsigos C. Stress hormones: physiological stress and regulation of metabolism. Curr Opin Pharmacol 2009;9:787-93.

3. Hamer M, Chida Y, Molloy GJ. Psychological distress and cancer mortality. J Psychosom Res 2009;66:255-8.

4. Nilsson MB, Sun H, Diao L, Tong P, Liu D, et al. Stress hormones promote EGFR inhibitor resistance in NSCLC: implications for combinations with $\beta$-blockers. Sci Transl Med 2017;9:eaao4307.

5. Nilsson MB, Armaiz-Pena G, Takahashi R, Lin YG, Trevino J, et al. Stress hormones regulate interleukin-6 expression by human ovarian carcinoma cells through a Src-dependent mechanism. J Biol Chem 2007;282:29919-26.

6. Vincent AS, Phan TT, Mukhopadhyay A, Lim HY, Halliwell B, et al. Human skin keloid fibroblasts display bioenergetics of cancer cells. J Invest Dermatol 2008;128:702-9.

7. Ghazizadeh M, Tosa M, Shimizu H, Hyakusoku H, Kawanami O. Functional implications of the IL-6 signaling pathway in keloid pathogenesis. J Invest Dermatol 2007;127:98-105.

8. Olaitan PB. Keloids: assessment of effects and psychosocial-impacts on subjects in a black African population. Indian J Dermatol Venereol Leprol 2009;75:368-72.

9. Furtado F, Hochman B, Farber PL, Muller MC, Hayashi LF, et al. Psychological stress as a risk factor for postoperative keloid recurrence. 
J Psychosom Res 2012;72:282-87.

10. Hochman B, Isoldi FC, Furtado F, Ferreira LM. New approach to the understanding of keloid: psychoneuroimmune-endocrine aspects. Clin Cosmet Investig Dermatol 2015;8:67-73.

11. Tan PL, Farmiloe S, Yeoman S, Watson JD. Expression of the interleukin 6 gene in rheumatoid synovial fibroblasts. J Rheumatol 1990;17:1608-12.

12. Feghali CA, Bost KL, Boulware DW, Levy LS. Control of IL-6 expression and response in fibroblasts from patients with systemic sclerosis. Autoimmunity 1994;17:309-18.

13. Gurram M, Pahwa S, Frieri M. Augmented interleukin-6 secretion in collagen-stimulated peripheral blood mononuclear cells from patients with systemic sclerosis. Ann Allergy 1994;73:493-96.

14. Shahar I, Fireman E, Topilsky M, Grief J, Kivity S, et al. Effect of IL-6 on alveolar fibroblast proliferation in interstitial lung diseases. Clin Immunol Immunopathol 1996;79:244-51.

15. Chistyakov DV, Astakhova AA, Sergeeva MG. Resolution of inflammation and mood disorders. Exp Mol Pathol 2018;105:190-201.

16. Doucet C, Brouty-Boye D, Pottin-Clemenceau C, Canonica GW, Jasmin C, et al. Interleukin (IL) 4 and IL-13 act on human lung fibroblasts. Implication in asthma. J Clin Invest 1998;101:2129-39.

17. Wynn TA. Fibrotic disease and the $\mathrm{T}(\mathrm{H}) 1 / \mathrm{T}(\mathrm{H}) 2$ paradigm. Nat Rev Immunol 2004;4:583-94.

18. Dasu MR, Hawkins HK, Barrow RE, Xue H, Herndon DN. Gene expression profiles from hypertrophic scar fibroblasts before and after IL-6 stimulation. J Pathol 2004;202:476-85.

19. Tosa M, Watanabe A, Ghazizadeh M. IL-6 polymorphism and susceptibility to keloid formation in a Japanese population. J Invest Dermatol 2016;136:1069-72.

20. Kiecolt-Glaser JK, Loving TJ, Stowell JR, Malarkey WB, Lemeshow S, et al. Hostile marital interactions, proinflammatory cytokine production, and wound healing. Arch Gen Psychiatry 2005;62:1377-84.

21. Glaser R, Robles TF, Sheridan J, Malarkey WB, Kiecolt-Glaser JK. Mild depressive symptoms are associated with amplified and prolonged inflammatory responses after influenza virus vaccination in older adults. Arch Gen Psychiatry 2003;60:1009-14.

22. Souza BR, Santos JS, Costa AM. Blockade of beta1- and beta2-adrenoceptors delays wound contraction and re-epithelialization in rats. Clin Exp Pharmacol Physiol 2006;33:421-30.

23. Pelat M, Verwaerde P, Galitzky J, Lafontan M, Berlan M, et al. High isoproterenol doses are required to activate beta3-adrenoceptormediated functions in dogs. J Pharmacol Exp Ther 2003;304:246-53.

24. de Mesquita CJG. About strawberry, crab claws, and the Sir James Black's invention. Hypothesis: can we battle keloids with propranolol? Med Hypotheses 2010;74:353-9.

25. Enoshiri T, Naitoh M, Yamawaki S, Kawaguchi A, Aya R, et al. $\beta$-adrenergic receptor blockers reduce the occurrence of keloids and hypertrophic scars after cardiac device implantation: a single-institution case-control study. Plast Reconstr Surg 2017;139:1248-56.

26. Herndon D, Capek KD, Ross E, Jay JW, Prasai A, et al. Reduced postburn hypertrophic scarring and improved physical recovery with yearlong administration of oxandrolone and propranolol. Ann Surg 2018;268:431-41.

27. Gonzalez-Cabrera PJ, Shi T, Yun J, McCune DF, Rorabaugh BR, et al. Differential regulation of the cell cycle by alpha1-adrenergic receptor subtypes. Endocrinology 2004;145:5157-67.

28. Lei B, Schwinn DA, Morris DP. Stimulation of alpha1 a adrenergic receptors induces cellular proliferation or antiproliferative hypertrophy dependent solely on agonist concentration. PLoS One 2013;8:e72430.

29. Sterin-Borda L, Furlan C, Orman B, Borda E. Differential regulation on human skin fibroblast by alpha1 adrenergic receptor subtypes. Biochem Pharmacol 2007;74:1401-12.

30. Waldrop BA, Mastalerz D, Piascik MT, Post GR. Alpha(1B)- and alpha(1D)-Adrenergic receptors exhibit different requirements for agonist and mitogen-activated protein kinase activation to regulate growth responses in rat 1 fibroblasts. J Pharmacol Exp Ther 2002;300:83-90.

31. Drummond PD, Dawson LF, Wood FM, Fear MW. Up-regulation of alpha1-adrenoceptors in burn and keloid scars. Burns 2018;44:582-8.

32. Zhang H, Faber JE. Trophic effect of norepinephrine on arterial intima-media and adventitia is augmented by injury and mediated by different alpha1-adrenoceptor subtypes. Circ Res 2001;89:815-22.

33. Faber JE, Szymeczek CL, Cotecchia S, Thomas SA, Tanoue A, et al. Alpha1-adrenoceptor-dependent vascular hypertrophy and remodeling in murine hypoxic pulmonary hypertension. Am J Physiol Heart Circ Physiol 2007;292:H2316-23.

34. Pang X, Sun NL. Calcineurin-NFAT signaling is involved in phenylephrine-induced vascular smooth muscle cell proliferation. Acta Pharmacol Sin 2009;30:537-44.

35. Zhang Q, Oh CK, Messadi DV, Duong HS, Kelly AP, et al. Hypoxia-induced HIF-1 alpha accumulation is augmented in a co-culture of keloid fibroblasts and human mast cells: involvement of ERK1/2 and PI-3K/Akt. Exp Cell Res 2006;312:145-55.

36. Zhao B, Guan H, Liu JQ, Zheng Z, Zhou Q, et al. Hypoxia drives the transition of human dermal fibroblasts to a myofibroblast-like phenotype via the TGF-beta1/Smad3 pathway. Int J Mol Med 2017;39:153-9.

37. Long F, Si L, Long X, Yang B, Wang XJ, et al. 2ME2 increase radiation-induced apoptosis of keloid fibroblasts by targeting HIF-1alpha in vitro. Australas J Dermatol 2016;57:e32-8.

38. Touchi R, Ueda K, Kurokawa N, Tsuji M. Central regions of keloids are severely ischaemic. J Plast Reconstr Aesthet Surg 2016;69:e35-41.

39. Zhang Z, Nie F, Kang C, Chen B, Qin Z, et al. Increased periostin expression affects the proliferation, collagen synthesis, migration and invasion of keloid fibroblasts under hypoxic conditions. Int J Mol Med 2014;34:253-61.

40. Jung YJ, Isaacs JS, Lee S, Trepel J, Neckers L. IL-1beta-mediated up-regulation of HIF-1alpha via an NFkappaB/COX-2 pathway identifies HIF-1 as a critical link between inflammation and oncogenesis. FASEB J 2003;17:2115-7. 
41. Karhausen J, Haase VH, Colgan SP. Inflammatory hypoxia: role of hypoxia-inducible factor. Cell Cycle 2005;4:256-8.

42. Cramer T, Johnson RS. A novel role for the hypoxia inducible transcription factor HIF-1alpha: critical regulation of inflammatory cell function. Cell Cycle 2003;2:192-3.

43. Okuno R, Ito Y, Eid N, Otsuki Y, Kondo Y, et al. Upregulation of autophagy and glycolysis markers in keloid hypoxic-zone fibroblasts: morphological characteristics and implications. Histol Histopathol 2018;33:1075-87.

44. Lee HJ, Jang YJ. Recent understandings of biology, prophylaxis and treatment strategies for hypertrophic scars and keloids. Int J Mol Sci 2018;19:711.

45. Jiao H, Fan J, Cai J, Pan B, Yan L, et al. Analysis of characteristics similar to autoimmune disease in keloid patients. Aesthetic Plast Surg $2015 ; 39: 818-25$

46. Gauglitz GG, Korting HC, Pavicic T, Ruzicka T, Jeschke MG. Hypertrophic scarring and keloids: pathomechanisms and current and emerging treatment strategies. Mol Med 2011;17:113-25.

47. Tredget EE, Nedelec B, Scott PG, Ghahary A. Hypertrophic scars, keloids, and contractures. The cellular and molecular basis for therapy. Surg Clin North Am 1997;77:701-30.

48. Chike-Obi CJ, Cole PD, Brissett AE. Keloids: pathogenesis, clinical features, and management. Semin Plast Surg 2009;23:178-84

49. Ogawa R. Keloid and hypertrophic scars are the result of chronic inflammation in the reticular dermis. Int J Mol Sci 2017;18:606.

50. Kiecolt-Glaser JK, Preacher KJ, MacCallum RC, Atkinson C, Malarkey WB, et al. Chronic stress and age-related increases in the proinflammatory cytokine IL-6. Proc Natl Acad Sci U S A 2003;100:9090-5.

51. Graham JE, Robles TF, Kiecolt-Glaser JK, Malarkey WB, Bissell MG, et al. Hostility and pain are related to inflammation in older adults. Brain Behav Immun 2006;20:389-400.

52. Webster Marketon JI, Glaser R. Stress hormones and immune function. Cell Immunol 2008;252:16-26.

53. Liu W, Wang DR, Cao YL. TGF-beta: a fibrotic factor in wound scarring and a potential target for anti-scarring gene therapy. Curr Gene Ther 2004;4:123-36.

54. Lee $\mathrm{CH}$, Hong $\mathrm{CH}$, Chen YT, Chen YC, Shen MR. TGF-beta1 increases cell rigidity by enhancing expression of smooth muscle actin: keloid-derived fibroblasts as a model for cellular mechanics. J Dermatol Sci 2012;67:173-80.

55. Tuan TL, Nichter LS. The molecular basis of keloid and hypertrophic scar formation. Mol Med Today 1998;4:19-24.

56. Ishihara H, Yoshimoto H, Fujioka M, Murakami R, Hirano A, et al. Keloid fibroblasts resist ceramide-induced apoptosis by overexpression of insulin-like growth factor I receptor. J Invest Dermatol 2000;115:1065-71.

57. Butler PD, Longaker MT, Yang GP. Current progress in keloid research and treatment. J Am Coll Surg 2008;206:731-41.

58. Ladak A, Tredget EE. Pathophysiology and management of the burn scar. Clin Plast Surg 2009;36:661-74.

59. Calcagni E, Elenkov I. Stress system activity, innate and T helper cytokines, and susceptibility to immune-related diseases. Ann N Y Acad Sci 2006;1069:62-76.

60. van den Broek LJ, van der Veer WM, de Jong EH, Gibbs S, Niessen FB. Suppressed inflammatory gene expression during human hypertrophic scar compared to normotrophic scar formation. Exp Dermatol 2015;24:623-9.

61. Namazi MR, Fallahzadeh MK, Schwartz RA. Strategies for prevention of scars: what can we learn from fetal skin? Int J Dermatol 2011;50:85-93.

62. Liechty KW, Kim HB, Adzick NS, Crombleholme TM. Fetal wound repair results in scar formation in interleukin-10-deficient mice in a syngeneic murine model of scarless fetal wound repair. J Pediatr Surg 2000;35:866-73.

63. Glaser R, Kennedy S, Lafuse WP, Bonneau RH, Speicher C, et al. Psychological stress-induced modulation of interleukin 2 receptor gene expression and interleukin 2 production in peripheral blood leukocytes. Arch Gen Psychiatry 1990;47:707-12.

64. Glaser R, MacCallum RC, Laskowski BF, Malarkey WB, Sheridan JF, et al. Evidence for a shift in the Th-1 to Th-2 cytokine response associated with chronic stress and aging. J Gerontol A Biol Sci Med Sci 2001;56:M477-82.

65. Mantovani A, Sica A, Sozzani S, Allavena P, Vecchi A, et al. The chemokine system in diverse forms of macrophage activation and polarization. Trends Immunol 2004;25:677-86.

66. Italiani P, Boraschi D. From monocytes to M1/M2 macrophages: phenotypical vs. functional differentiation. Front Immunol $2014 ; 5: 514$.

67. Mills CD, Ley K. M1 and M2 macrophages: the chicken and the egg of immunity. J Innate Immun 2014;6:716-26.

68. Jin Q, Gui L, Niu F, Yu B, Lauda N, et al. Macrophages in keloid are potent at promoting the differentiation and function of regulatory T cells. Exp Cell Res 2018;362:472-6.

69. Schmidt A, Oberle N, Krammer PH. Molecular mechanisms of treg-mediated T cell suppression. Front Immunol 2012;3:51.

70. Dey A, Hankey Giblin PA. Insights into macrophage heterogeneity and cytokine-induced neuroinflammation in major depressive disorder. Pharmaceuticals (Basel) 2018;11:64.

71. Chen X, Thibeault SL. Role of tumor necrosis factor-alpha in wound repair in human vocal fold fibroblasts. Laryngoscope 2010;120:1819-25.

72. Haapakoski R, Mathieu J, Ebmeier KP, Alenius H, Kivimaki M. Cumulative meta-analysis of interleukins 6 and 1beta, tumour necrosis factor alpha and C-reactive protein in patients with major depressive disorder. Brain Behav Immun 2015;49:206-15.

73. Kohler CA, Freitas TH, Stubbs B, Maes M, Solmi M, et al. Peripheral alterations in cytokine and chemokine levels after antidepressant drug treatment for major depressive disorder: systematic review and meta-analysis. Mol Neurobiol 2018;55:4195-206. 